\title{
Are Peripheral Blood Counts Predictor of Bone Marrow Infiltration and Hypocellularity in Malignant Neoplasms?
}

\author{
Sobia Ashraf, Ambareen Hamid, Samina Qamar, Hajirah Syndeed Pal, Qurat-ul-Ain and Sindhu Rehman \\ Department of Pathology, King Edward Medical University, Lahore, Pakistan
}

\begin{abstract}
Objective: To determine the association of peripheral blood counts (cytopenia and normal counts) with bone marrow findings in patients with malignant neoplasms.

Study Design: Descriptive study.

Place and Duration of the Study: King Edward Medical University from 2017 to 2019.

Methodology: Patients having malignant diseases, of both gender and all ages, were included. Clinical data and results of bilateral bone marrow aspiration and biopsy were noted. Results were categorised as groups with bone marrow infiltration, normal morphology with normal cellularity and normal morphology with decreased cellularity. Results were analysed using SPSS V21 applying Chi-square test, keeping the confidence interval $95 \%$ and $p$-value of $<0.05$ as significant.

Results: A total of 157 patients were included (107 men, 50 women). The main categories of neoplasm included non-Hodgkin's lymphoma [93 (59.2\%)] Hodgkin's lymphoma [31 (19.7\%)], and solid organ malignancy [33 (21.0\%]. Bone marrow infiltration was seen in $50(31.8 \%)$ patients, normal morphology with decreased cellularity in $51(32.5 \%)$ and normal morphology with normal cellularity in $56(35.7 \%)$ patients. Cytopenias were seen in $88(56.1 \%)$ patients (out of these $28(31.8 \%)$ had bone marrow infiltrarion, $32(36.4 \%)$ had decreased cellularity and 28 (31.8\%) with normal cellularity). In $69(43.9 \%)$ patients with normal CBC, 22 (31.9\%) had infiltration, 19 (27.5\%) had decreased cellularity and 28 (40.6\%) had normal cellularity. No association of cytopenia with bone marrow results was obtained $(p=0.086)$.

Conclusion: Bone marrow infiltration and hypocellularity are not reflected by peripheral blood counts. Therefore, bone marrow biopsy remains decisive in patients with malignant neoplasm for disease staging and selection of treatment options.
\end{abstract}

Key Words: Peripheral blood counts, Bone marrow infiltration, malignant neoplasms, bone marrow hypocellularity.

How to cite this article: Ashraf S, Hamid A, Qamar S, Pal HS, QUA, Rehman S. Are Peripheral Blood Counts Predictor of Bone Marrow Infiltration and Hypocellularity in Malignant Neoplasms?. J Coll Physicians Surg Pak 2021; 31(01):79-82.

\section{INTRODUCTION}

Bone marrow biopsy is an important procedure to assess the hemopoietic activity. Bone marrow activity along with many other factors affect the peripheral blood counts. Cytopenia is the term given when any of the cell line is decreased (anemia, thrombocytopenia or leucopenia alone or in combination). ${ }^{1}$ Bone marrow aspirate yields information regarding morphology of cells, percentage population and presence of abnormal cells. Bone marrow trephine biopsy is a useful tool for determining the morphological relationships between cells, bone, bone marrow stroma, cellularity and normal and abnormal cellular distributions and infiltration, if present. $^{2}$

Correspondence to: Dr. Sobia Ashraf, Department of Pathology, King Edward Medical University, Lahore, Pakistan

E-mail: s.qayyum81@gmail.com

Received: February 26, 2020; Revised: June 13, 2020;

Accepted: July 20, 2020

DOI: https://doi.org/10.29271/jcpsp.2021.01.79
Malignant neoplasms are an important cause of morbidity and mortality throughout the world. In 2018, according to World Cancer Registry, eight million cases were observed. ${ }^{3}$ Malignant neoplasms can be broadly divided into lymphoid and non-lymphoid neoplasms. Lymphomas usually arise in lymph nodes and/or secondary lymphoid organs. Bone marrow can be infiltrated by these malignant neoplasms. This infiltration affects normal cellular production and can lead to cytopenias. ${ }^{4}$

Bone marrow is also affected by systemic effects of cytokines produced by the body in response to tumor. Tumor necrosis factor alpha (TNF $\alpha$ ) and interferon gamma (IFN $N_{Y}$ ) have proven role in suppression of hemopoietic cells. These along with other cytokines cause suppression of the normal hemopoietic activity, and can lead to cytopenias. ${ }^{5-7}$ Nutritional deficiencies, infections and autoimmune phenomenon are also contributory factors in these morbid conditions. ${ }^{8}$

Few lymphomas arise primarily in the bone marrow like hairy cell Leukemia, chronic lymphocytic leukemia etc. For those arising in other tissues, bone marrow biopsy is performed for staging purposes. According to Ann Arbor classification, lymphoma is classified as stage 4 if bone marrow infiltration is 
present. This staging affects treatment protocol as radiation therapy plus chemotherapy is given in stage 1 and 2 , and increased cycles of chemotherapy without radiation in stage 3 and $4 .^{9}$

Bone marrow biopsy in non-lymphoid neoplasms in presence of cytopenias is performed to rule out bone marrow infiltration. However bone marrow infiltration may even be seen with normal peripheral counts. ${ }^{9}$ Bone marrow findings with peripheral blood in the form of infiltration, decreased cellularity and nutritional deficiency, can help the clinician in making decisions regarding supportive and therapeutic treatment protocols. Few studies have recommended PET scan instead. However, bone marrow biopsy is still superior due to additional information obtained from this test as mentioned above. ${ }^{10}$

The objective of this study was to determine frequency of cytopenias in patients with malignant neoplasms referred for bone marrow biopsy and association of peripheral blood counts (cytopenias and normal blood counts) with bone marrow findings in these patients.

\section{METHODOLOGY}

This descriptive study was conducted in Pathology Department, King Edward Medical University Lahore from 2017 to 2019 after taking approval from the Ethical Research Committee. Patients of both genders and all ages with established primary diagnosis of malignant neoplasm referred from the Medical Wards and Oncology Department of Mayo Hospital were included, while patients with primary disease of bone marrow, those on treatment for malignancy and having secondary malignancy were excluded.

Slides of bilateral bone marrow aspiration and paraffin embedded trephine biopsy sections were reviewed for cellularity and infiltration. Bone marrow biopsy results were categorised as Infiltration and without infiltration. Those without infiltration were assessed for cellularity and categorised as normal morphology with decreased cellularity and normal morphology with normal cellularity. Cellularity was calculated subjectively, and according to the age of the patient. Data was entered and analysed on SPSS version 21. Qualitative variables like gender, age, bone marrow diagnosis, and cellularity were expressed as percentages and frequencies and Chi-square test was applied as test of significance, keeping the confidence interval of $95 \%$ and $p$ value of $\leq 0.05$.

\section{RESULTS}

A total of 157 patients were included in the study with 107 $(68.2 \%)$ men and 50 (31.8\%) women. Out of these 157 patients, $93(59.2 \%)$ patients including 65 (69.9\%) men and 28 (30.1\%) women had non-Hodgkin's lymphoma as the primary diagnosis; 31 (19.7\%) patients 20 (64.5\%) men and 11 (35.5\%) women) had Hodgkin's lymphoma; and $33(21.0 \%)$ patients 22 (66.7\%) men and 11 (33.3\%) women) presented with solid organ malignancy. The age of the patients varied from 1 to 80 years. NHL was more common in 51-60 years age group, Hodgkin's lymphoma in 21-30 and 41-50 age group, while solid organ malignancy cases were mostly of retinoblastoma and this was more prevalent in 1-10yearage group.

Cytopenias were observed as either single, bicytopenia or pancytopenia (Tablel).

Out of the 157 patients, 50 (31.8\%) patients had infiltration, 51 $(32.5 \%)$ patients had decreased cellularity for age of the patient and 56 (35.7\%) patients had normal cellularity. Association of cytopenia with bone marrow infiltration, decreased cellularity and normal marrow was determined (Table II) and no association wasseen ( $p=0.086$, Table III).

Table I: Frequency of cytopenias.

\begin{tabular}{|l|c|c|}
\hline Cytopenias & Frequency & Percentage \% \\
\hline None & 69 & 43.9 \\
\hline Anemia & 53 & 33.8 \\
\hline Thrmbocytopenia & 5 & 3.2 \\
\hline Leukopenia & 1 & 0.6 \\
\hline Anemia and thrmbocytopenia & 15 & 9.6 \\
\hline Anemia and leukopenia & 6 & 3.8 \\
\hline Pancytopenia & 8 & 5.1 \\
\hline Total & 157 & 100.0 \\
\hline
\end{tabular}

\section{DISCUSSION}

In this study, 157 patients were included; 93 (59.2\%) patients had NHL, being more prevalent in males. Study conducted by Horesh et al. also reported male predominance due to protective effects of female sex hormone, pregnancy, live births and use of oral contraceptives. ${ }^{11}$

In 93 patients of $\mathrm{NHL}$, bone marrow infiltration was seen in 32 (34.4\%) patients; while 61 patients had normal morphology bone marrow with decreased celluarity (32 patients) and normal cellularity (31 patients). Results of study conducted by Sultan et al. (31.5\%) are in accordance with the present study. ${ }^{4}$ However, this frequency of bone marrow infiltration varies from places to places and has been found to be $27 \%$ in Australia, $61.4 \%$ in Israel, $32 \%$ in USA, and $33.8 \%$ in Croatia. ${ }^{12}$

In NHL patients, cytopenias were seen in 47 (50.54\%) patients, while $46(49.46 \%)$ patients had normal peripheral counts. Among the patients with cytopenias $22(46.8 \%)$ patients had bone marrow infiltration, 13 (27.66) had decreased cellularity and $12(25.53 \%)$ had normal cellularity. In patients with normal counts 10 (21.73\%) had bone marrow infiltration, 17 (36.95\%) had decreased cellularity and $19(41.3 \%)$ had normal cellularity. This data suggests that one of the important causes of cytopenia is decreased cellularity of bone marrow. However, bone marrow infiltration can be seen even with normal counts. This was also highlighted by Lim et al., who saw $66 \%$ patients having anemia and marrow infiltration in $46 \%$ patients. ${ }^{13} \mathrm{~A}$ study by H. Soliman attributed marrow hypocellularity and a high International prognostic index (IPI) $(p<0.05)$ to raised levels of TNF $\alpha$ and L-selectin in NHL patients leading to increased BM reticular cells, macrophages and fibroblasts. ${ }^{14}$ 
Table II: Bone marrow diagnosis and peripheral blood counts.

\begin{tabular}{|c|c|c|c|c|c|c|}
\hline \multirow{2}{*}{ Diagnosis } & \multirow{2}{*}{ Cytopenia } & \multirow[b]{2}{*}{ Infiltration } & \multicolumn{3}{|c|}{ Bone marrow diagnosis } & \multirow{2}{*}{ p-value } \\
\hline & & & NMM dec cellularity & NMM with NC & Total & \\
\hline \multirow{5}{*}{$\mathrm{NHL}$} & None & $10(31.25 \%)$ & $17(56.67 \%)$ & $19(61.29 \%)$ & $46(49.46 \%)$ & \multirow{5}{*}{0.223} \\
\hline & Single cytopenia & $12(37.5 \%)$ & $10(33.33 \%)$ & $8(25.81 \%)$ & $30(32.26 \%)$ & \\
\hline & Bicytopenia & $6(18.75 \%)$ & $3(10.0 \%)$ & $3(9.68 \%)$ & $12(12.89 \%)$ & \\
\hline & Pancytopenia & $4(12.5 \%)$ & $0(0 \%)$ & $1(3.23 \%)$ & $5(5.37 \%)$ & \\
\hline & Total & $32(100 \%)$ & $30(100 \%)$ & $31(100 \%)$ & $93(100 \%)$ & \\
\hline \multirow{5}{*}{$\mathrm{HL}$} & None & $4(80.0 \%)$ & $4(57.14 \%)$ & $8(42.11 \%)$ & $16(51.61 \%)$ & \multirow{5}{*}{0.419} \\
\hline & Single cytopenia & $0(0 \%)$ & $3(42.86 \%)$ & $9(47.37 \%)$ & $12(38.71 \%)$ & \\
\hline & Bicytopenia & $0(0 \%)$ & $0(0 \%)$ & $1(5.26 \%)$ & $1(3.23 \%)$ & \\
\hline & Pancytopenia & $1(20 \%)$ & $0(0 \%)$ & $1(5.26 \%)$ & $2(6.45 \%)$ & \\
\hline & Total & $5(100 \%)$ & $7(100 \%)$ & $19(100 \%)$ & $31(100 \%)$ & \\
\hline \multirow{5}{*}{$\begin{array}{l}\text { Solid organ } \\
\text { malignancy }\end{array}$} & None & $3(23.08 \%)$ & $3(21.43 \%)$ & $3(50 \%)$ & $9(27.27 \%)$ & \multirow{5}{*}{0.194} \\
\hline & Single cytopenia & $6(46.15 \%)$ & $9(64.29 \%)$ & $3(50 \%)$ & $18(54.54 \%)$ & \\
\hline & Bicytopenia & $3(23.08 \%)$ & $2(14.29 \%)$ & $0(0 \%)$ & $5(15.15 \%)$ & \\
\hline & Pancytopenia & $1(7.69 \%)$ & $0(0 \%)$ & $0(0 \%)$ & $1(3.03 \%)$ & \\
\hline & Total & $13(100 \%)$ & $14(100 \%)$ & $6(100 \%)$ & $33(100 \%)$ & \\
\hline
\end{tabular}

Table III: Association of various cytopenias.

\begin{tabular}{|c|c|c|c|c|c|c|}
\hline & & Infiltration & $\begin{array}{l}\text { NMM dec } \\
\text { cellularity }\end{array}$ & NMM with NC & Total & $\begin{array}{c}\mathbf{p} \\
\text { value }\end{array}$ \\
\hline \multirow[t]{7}{*}{ Name of cytopenia } & None & $17(34 \%)$ & $24(47.06 \%)$ & $30(53.57 \%)$ & $71(45.22 \%)$ & 0.032 \\
\hline & Anemia & $15(30.0 \%)$ & $20(39.22 \%)$ & $18(32.14 \%)$ & $53(33.76 \%)$ & \\
\hline & Thrombocytopenia & $3(6.0 \%)$ & $1(1.96 \%)$ & $2(3.57 \%)$ & $6(3.82 \%)$ & \\
\hline & Leukopenia & $0(0 \%)$ & $1(1.96 \%)$ & $0(0 \%)$ & $1(0.6 \%)$ & \\
\hline & Anemia and thrombocytopenia & $9(18 \%)$ & $3(5.88 \%)$ & $2(3.57 \%)$ & $14(8.92 \%)$ & \\
\hline & Anemia and leukopenia & $0(0 \%)$ & $2(3.92 \%)$ & $2(3.57 \%)$ & $4(2.55 \%)$ & \\
\hline & Pancytopenia & $6(12 \%)$ & $0(0 \%)$ & $2(3.57 \%)$ & $8(5.10 \%)$ & \\
\hline Total & & $50(100 \%)$ & $51(100 \%)$ & $56(100 \%)$ & $157(100 \%)$ & \\
\hline
\end{tabular}

In 31 patients of Hodgkin's lymphoma, infiltration was seen in 5 (16.1\%) patients. It is almost in accordance with the study by Lone et al. In our study, cytopenias were seen in 15 patients. Out of these 15 patients, cytopenias were seen with bone marrow infiltration in $1(6.67 \%)$ patient, $3(20 \%)$ patients had cytopenias due to decreased cellularity of bone marrow and 11 (73.33\%) patients had cytopenias with normal morphology bone marrow. In a study conducted by Hohaus et al., anemia was seen in $40 \%$ of the patients and this was associated with raised levels of Hepcidin, which is stimulated by TNF and other cytokines. In a recent study by Linaburg et al, increased incidence of aplastic anemia was found to be associated with Hodgkin's disease and most probable etiology was immune dysregulation. ${ }^{15}$ Similar mechanisms are involved in inducing marrow hypocellularity.

In solid organ malignancy, majority of the cases were of round blue cell tumor. Infiltration was seen in 13 out of 33 patients (39.39\%), decreased cellularity in 14 patients $(42.4 \%)$ and normal cellularity in $6(18.2 \%)$ patients. Cytopenias were observed in 24 out of $33(72.7 \%)$ patients; $10(41.7 \%)$ of cytopenic patients had infiltration, while 11 (45.8\%) patients had decreased cellularity and $3(12.5 \%)$ patients had normal cellularity. This data suggests that bone marrow suppression in the form of decreased cellularity is more pronounced in these neoplasms. A study conducted by Rastogi et al. showed $54 \%$ marrow involvement in neuroblastoma, while anemia was seen both in involved and uninvolved cases, 39\% patients had anemia and thrombocytopenia without bone marrow infiltration. ${ }^{16}$

TNF $\alpha$, IFN ${ }_{\gamma}$ and other cytokines have established inhibitory effects on hemopoietic progenitor cells. TNF has contributory role in raising levels of hepcidin. Hepcidin is a key protein in anemia of chronic disease. A study conducted by Atinkson et al. described rise in hemoglobin and fall in hepcidin levels after anti-TNF-therapy. ${ }^{17}$

No significant association was found in low peripheral blood counts and marrow involvement (infiltration / hypo cellularity); still the morphological findings of bone marrow biopsy are decisive in the management of patients regarding treatment protocols, transfusion requirements and frequency of follow-up visits.

\section{CONCLUSION}

Peripheral blood cytopenias are not a predictor of bone marrow morphological findings.

Bone marrow biopsy remains a highly effective tool in malignant neoplasm management. It provides information about staging of the disease and haemopoetic activity of bone marrow; thus enabling the treating clinician selecting appropriate treatment options for the patients. 


\section{ETHICAL APPROVAL:}

Ethical approval of this study was obtained from King Edward Medical University Ethics Committee prior to initiation of the research work (Vide Letter No. 2183/RC/KEMU).

\section{PATIENTS' CONSENT:}

Informed consents were obtained from all patients to publish the data concerning this study.

\section{CONFLICT OF INTEREST:}

Authors declared no conflict of interest.

\section{AUTHORS' CONTRIBUTION:}

$\mathrm{SA}, \mathrm{AH}$ : Conception and design of the study, drafting and final approval, agreement to be accountable for all aspects of the work in ensuring that questions related to the accuracy or integrity of any part of the work are appropriately investigated and resolved. SQ: Drafting the study, acquisition of data and final approval. HSP: Drafting the study, data analysis and interpretation. QA: Conception of the work and final approval of the study. SR: Drafting the study, data acquisition and interpretation.

\section{REFERENCES}

1. Hoffbrand AV, Pettit JE, Moss PAH. (2015). Essential haemato-logy. ISBN: 978-1-118-40867-4 October 2015 Oxford: Blackwell Science.

2. Bain B, Bates I, Laffan M, Dacie and Lewis Practical Haemato-logy. ISBN: 9780702066962 September 2016; Elsevier.

3. Ferley J, Colombet $M$, Soerjomataram I, Mathers C, Parkin $D M$, Pineros M. Estimating the global cancer incidence and mortality in 2018: GLOBOCAN sources and methods. Int J Cancer 2019; 144(8):1941-53. doi: 10.1002/ijc. 31937.

4. Sultan S, Baloch N, Ahmed ZA, Irfan SM, Parveen S. Pattern of bone marrow involvement in non-Hodgkin's lymphoma classified according to WHO classification: Report of a developing country Pakistan. J Lab Physicians 2018; 10(1):17-20. doi: 10.4103/JLP.JLP_9_17.

5. Broxmeyer HE, Williams DE, Lu L, Cooper S, Anderson SL, Beyer GS, et al. The suppressive influences of human tumor necrosis factors on bone marrow hematopoietic progenitor cells from normal donors and patients with leukemia: Synergism of tumor necrosis factor and interferon-gamma. J Immunol 1986; 136 (12):4487-95.

6. Papadaki HA, Kritikos HD, Gemetzi C, Koutala H, Marsh CW, Boumpas DT, et al. Bone marrow progenitor cell reserve and function and stromal cell function are defective in rheumatoid arthritis: Evidence for a tumor necrosis factor alpha-mediated effect. Blood 2002; 99(5):1610-19. doi: http://doi.org/ 10.1182/blood.V99.5.1610.
7. Warzocha K, Salles G, Bienvenu J, Bastion Y, Dumontet C, Renard N, et al. Tumor necrosis factor ligand-receptor system can predict treatment outcome in lymphoma patients. J Clin Oncol 1997; 15(2):499-508. doi: 10.1200/JCO.1997.15.2.499.

8. Kaur K, Sharma N, Gupta K, Gulati S, Choudhary P. Hematological and Bone Marrow Biopsy Evaluation in Non-Hodgkin Lymphoma. Int J Cur Res Rev 2017; 9(3).

9. Barbara J. Bain, David M. Clark, Bridget S. Wilkins. Bone Marrow Pathology, John Willey \&sons, Hoboken, USA. 2019.

10. Alzahrani M, Galaly $T$, Hutchings $M$, Hansen J, Loft $A$, Johnsen $\mathrm{HE}$, et al. The value of routine bone marrow biopsy in patients with diffuse large B-cell lymphoma staged with PET/CT: A Danish-Canadian study. Ann Oncol 2016; 27(6):1095-9. doi: 10.1093/annonc/mdw137.

11. Horesh N, Horowitz NA. Does gender matter in nonhodgkin lymphoma? Differences in epidemiology, clinical behavior, and therapy. Rambam Maimonides Med J 2014; 5(4): e0038. http://doi.org/10.5041/RMMJ.10172.

12. Ishtiaq J, Jehanzeb K, Ali N, Alam M. Bone marrow infiltration and patterns in previously diagnosed patients of nonhodgkin lymphoma (a single centre study). Pak Armed Forces Med J 2017; 67(2):249-5. pafmj.org/ index.php/PAFMJ/article/view/364.

13. Lim EJ, Peh SC. Bone marrow and peripheral blood changes in non-hodgkin's lymphoma Singapore. Med J 2000; 41(6):279-85.

14. Soliman AH. Diagnostic and prognostic relevance of bone marrow microenvironment components in non-Hodgkin's lymphoma cases before and after therapy. Asian Pac J Cancer Prev 2016; 17(12):5273-80. doi:10.22034/ APJCP.2016.17.12.5273.

15. Linaburg T, Davis AR, Frey NV, Khawaja MR, Landsburg DJ, Schuster SJ, et al. Hodgkin lymphoma patients have an increased incidence of idiopathic acquired aplastic anemia. PLos One 2019; 14(4):e0215021. doi: 10.1371/journal.pone.0215021.

16. Rastogi P, Naseem S, Varma N, Das R, Ahluwalia J, Sharma $P$, et al. Bone marrow involvement in neuroblastoma: $A$ study of hemato-morphological features. Indian J Hematol Blood Transfus 2015; 31(1):57-60. doi: 10.1007/ s12288014-0405-1.

17. Atkinson MA, Leonard MB, Herskovitz R, Baldassano RN, Denburg MR. Changes in hepcidin and hemoglobin after anti-tnf-alpha therapy in children and adolescents with crohn disease. J Pediatr Gastroenterol Nutr 2018; 66(1):90-4. doi:10.1097/MPG.0000000000001650. 